\title{
FULL REGULARITY OF BOUNDED SOLUTIONS TO NONDIAGONAL PARABOLIC SYSTEMS OF TWO EQUATIONS
}

Abstract. Hölder continuity and, basing on this, full regularity and global existence of weak solutions is studied for a general nondiagonal parabolic system of nonlinear differential equations with the matrix of coefficients satisfying special structure conditions and depending on the unknowns. A technique based on estimating a certain function of unknowns is employed to this end.

1. Introduction. In the present paper we study the Hölder continuity, full regularity and global existence of solutions to a nonlinear nondiagonal parabolic system of two equations in divergence form under special assumptions upon its structure.

It is well-known that the De Giorgi-Nash-Moser estimates are no longer valid in general for an elliptic system; the latter can be regarded as a special case of the parabolic version. An example of an unbounded solution to a linear elliptic system with bounded coefficients was built up by De Giorgi in [4]. There is yet another example due to J. Nečas and J. Souček of a nonlinear elliptic system with coefficients sufficiently smooth, but with a weak solution not belonging to $W^{2,2}$.

These two and many other examples illustrate that the regularity problem for elliptic systems proves to be far more complicated than that for second order elliptic equations.

For systems of differential equations, until now a priori estimates of De Giorgi type have been extended only to a special class of parabolic systems of equations, the so-called weakly coupled systems.

2000 Mathematics Subject Classification: Primary 35B50; Secondary 35K40, 35K55.

Key words and phrases: nondiagonal parabolic systems, Hölder continuity, Dirichlet data. 
Therefore the question of finding strongly coupled systems whose solutions exhibit a certain regularity is of interest.

The technique we are utilizing has been employed earlier in [10] for semilinear systems (see also [6], [11] and [7]), and consists in switching to new functions, for which the estimate is established in a conventional way, whence the final conclusion about each component of the vector function solution follows. This technique allows one to tackle nonlinear nondiagonal systems.

The main idea of our approach is as follows: instead of trying to establish estimates for each component of a solution $(u, v)$ we introduce some new functions $H(u, v)$ of the components from whose estimates we shall be able to derive estimates for the components themselves.

In the present paper, although restricting ourselves to systems of second order equations in divergence form possessing a special structure, we demonstrate full regularity of solutions to nonlinear parabolic systems of equations in which coupling occurs in the leading derivatives and whose leading coefficients depend on $x, u$, and $v$.

For our results it is crucial that we assume the $L^{\infty}$ boundedness of solutions to system (2.1). Some results on boundedness of solutions to system (2.1) under conditions (2.3)-(2.9) will appear elsewhere.

2. Basic notations and hypotheses. We shall be concerned with a system of two equations of the form

$$
\left\{\begin{array}{l}
u_{t}-\frac{\partial}{\partial x_{i}}\left(a_{1}(x, u, v) \nabla u+b_{1}(x, u, v) \nabla v\right)=f_{1}(x, t) \frac{1}{\sqrt{1+|u|+|v|}} \\
v_{t}-\frac{\partial}{\partial x_{i}}\left(a_{2}(x, u, v) \nabla u+b_{2}(x, u, v) \nabla v\right)=f_{2}(x, t) \frac{1}{\sqrt{1+|u|+|v|}} \\
(x, t) \in Q \\
f_{j}(x, t) \in L^{\tau}(Q), \quad \tau=\frac{2+n}{2-n \kappa}, \quad \kappa \in(0,2 / n)
\end{array}\right.
$$

Moreover we suppose that there are two positive linearly independent functions of two variables $H_{1}(u, v)>0$ and $H_{2}(u, v)>0$ (we shall write $H$ for both $H_{1}$ and $H_{2}$ ) such that for all $u, v, x \in \mathbb{R}$,

$$
\begin{gathered}
C_{1}\left(u^{2}+v^{2}\right)-K \leq H(u, v) \leq C_{2}\left(u^{2}+v^{2}\right)+K, \\
0 \leq\left|H_{u}(u, v)\right|,\left|H_{v}(u, v)\right| \leq C_{2}(|u|+|v|)+K, \\
C_{1} \leq\left|H_{u u}(u, v)\right|,\left|H_{u v}(u, v)\right|,\left|H_{v v}(u, v)\right| \leq C_{2},
\end{gathered}
$$

where $0<C_{1}, C_{2}, K<\infty$ are constants, and the following hypotheses hold:

$$
\left\{\begin{array}{l}
a_{1}(x, u, v) H_{u}(u, v)+a_{2}(x, u, v) H_{v}(u, v)=\Lambda(x, u, v) H_{u}(u, v), \\
b_{1}(x, u, v) H_{u}(u, v)+b_{2}(x, u, v) H_{v}(u, v)=\Lambda(x, u, v) H_{v}(u, v)
\end{array}\right.
$$


and

$$
\left\|\begin{array}{cc}
a_{1} H_{u u}+a_{2} H_{u v} \geq 0 \\
2\left(a_{1} H_{u u}+a_{2} H_{u v}\right) & \left(a_{1}+b_{2}\right) H_{u v}+b_{1} H_{u u}+a_{2} H_{v v} \\
\left(a_{1}+b_{2}\right) H_{u v}+b_{1} H_{u u}+a_{2} H_{v v} & 2\left(b_{1} H_{u v}+b_{2} H_{v v}\right)
\end{array}\right\| \geq 0,
$$

where $\Lambda: \Omega \times \mathbb{R} \times \mathbb{R} \rightarrow \mathbb{R}$ is a measurable function such that

$$
0<\Lambda_{1} \leq \Lambda(x, u, v) \leq \Lambda_{2}, \quad \forall u, v, x \in \mathbb{R},
$$

for some numbers $\Lambda_{1,2}$. The inverse functions $u=H_{1}^{-1}\left(H_{1}, H_{2}\right)$ and $v=H_{2}^{-1}\left(H_{1}, H_{2}\right)$ are continuously differentiable:

$$
H_{1}^{-1}, H_{2}^{-1} \in C^{1}(\mathbb{R}, \mathbb{R}) .
$$

Additionally we assume that the coefficients $a_{1}, a_{2}, b_{1}, b_{2}$ satisfy an ellipticity condition (the strong Legendre condition) and are twice continuously differentiable functions of $u$ and $v$ :

$$
a_{1}(x, u, v), a_{2}(x, u, v), b_{1}(x, u, v), b_{2}(x, u, v) \in C^{2}(\cdot, \mathbb{R}, \mathbb{R}) .
$$

EXAMPLE. Here is an example of a parabolic model system satisfying our hypotheses:

$$
\begin{gathered}
a_{1}(u, v)=\Lambda_{1}(u, v)-H_{2 u} H_{1 v} \eta(u, v) ; \quad a_{2}(u, v)=H_{1 u} H_{2 u} \eta(u, v) \\
b_{1}(u, v)=-H_{2 v} H_{1 v} \eta(u, v) ; \quad b_{2}(u, v)=\Lambda_{1}(u, v)+H_{1 u} H_{2 v} \eta(u, v) \\
|\eta(u, v)|=C_{3} /\left(1+\left|k_{1}\right|+\left|k_{2}\right|+u^{4}+v^{4}\right) \\
H_{1}(u, v)=\delta\left(u^{2}+v^{2}+\varepsilon u v\right)+u+k_{1} v ; \\
H_{2}(u, v)=\delta\left(u^{2}+v^{2}+\varepsilon u v\right)+u+k_{2} v ; \\
C_{1} \leq \Lambda_{1}(u, v) \leq C_{2} ; \quad|\varepsilon| \leq 1 / 10 ; \quad C_{1} \geq 5 C_{3}>0 ; \\
0<\delta<\max [1 ; 1 /(4 M)] ; \quad \delta, \varepsilon, k_{1}, k_{2}=\text { const; } \quad k_{1}, k_{2}>0 ; \quad k_{1} \neq k_{2} .
\end{gathered}
$$

The boundary conditions of the Dirichlet type are assigned:

$$
\left\{\begin{array}{l}
\left(u-g_{1}, v-g_{2}\right)(x, t) \in W_{0}^{1,2}(\Omega) \quad \text { a.e. } t \in(0, T), \\
(u, v)(x, 0)=\left(u_{0}, v_{0}\right)(x) .
\end{array}\right.
$$

A positive solution to system (2.1) with Dirichlet data (2.12) is understood in the weak sense, as in [5].

Let us describe the notions, quantities and functions entering system (2.1) that will appear in this paper.

Here and onward we use the following notations: $Q=\Omega \times(0, T] ; S=$ $\partial \Omega \times(0, T] ; \partial Q \equiv\{\Omega \times\{0\}\} \cup\{\partial \Omega \times(0, T]\} ; \partial Q^{\prime}$ is a portion of $\partial Q ;$ $\Omega$ is a bounded domain in $\mathbb{R}^{n}$ with piecewise smooth boundary; $x \in \Omega$; $T>0 ; t \in(0, T] ; n \geq 2 ; i=1, \ldots, n ; j=1,2$ and summation convention over repeated indices is assumed; $u, v \in C\left(0, T ; L^{2}(\Omega)\right) \cap L^{2}\left(0, T ; W^{1,2}(\Omega)\right)$; 
$W_{0}^{1,2}(\Omega)$ is the space of functions in $W^{1,2}(\Omega)$ vanishing on $\partial \Omega$ in the sense of traces for a.e. $t \in(0, T]$.

The boundary $\partial \Omega$ of the domain $\Omega$ is assumed to satisfy condition $(A)$ : there are positive numbers $a_{0}$ and $\theta_{0}$ such that for any ball $K_{\varrho}$ with center on $\partial \Omega$ and radius $\varrho \leq a_{0}$,

$$
\operatorname{mes} K_{\varrho} \cap \Omega \leq\left(1-\theta_{0}\right) \operatorname{mes} K_{\varrho} .
$$

By parabolicity of system (2.1) it is meant that the part without derivatives with respect to time is elliptic. As the definition of ellipticity of a system of differential equations we take the strong Legendre condition.

Assume that

$$
\left|a_{j}(x, r)\right|,\left|b_{j}(x, r)\right| \leq \Lambda_{2}, \quad \forall r \in \mathbb{R}^{2}, \forall x \in \mathbb{R}^{n} .
$$

For simplicity we write

$$
\begin{aligned}
& \widetilde{u}_{0}= \begin{cases}u_{0}(x), & x \in \Omega, t=0, \\
g_{1}(x, t), & x \in \partial \Omega, t \in(0, T),\end{cases} \\
& \widetilde{v}_{0}= \begin{cases}v_{0}(x), & x \in \Omega, t=0, \\
g_{2}(x, t), & x \in \partial \Omega, t \in(0, T) .\end{cases}
\end{aligned}
$$

Let $m$ be a nonnegative integer, $\alpha \in(0,1)$ and $a=m+\alpha$. We put

$$
|u|_{a ; Q}=\sum_{\nu+2 \mu \leq m} \sup _{Q}\left|D_{x}^{\nu} D_{t}^{\mu} u\right|+\sum_{\nu+2 \mu=m} \sup _{Q}\left[D_{x}^{\nu} D_{t}^{\mu} u\right]_{\alpha ; Q},
$$

where $[\ldots]_{\alpha ; Q}$ stands for the Hölder norm and $u \in B C^{a, a / 2}(Q)$ if $|u|_{a ; Q}<\infty$.

The functions $g_{j}(x, t),\left(u_{0}, v_{0}\right)(x)$ in boundary data $(2.12)$ are assumed to satisfy

$$
g_{j} \in B C^{\alpha_{g}, \alpha_{g} / 2}(S), \quad u_{0}, v_{0} \in B C^{\alpha_{0}}(\bar{\Omega} \times\{0\})
$$

with $\alpha_{g} \in(0,1)$ and $\alpha_{0} \in(0,1)$.

We also assume that the components $(u, v)$ of the solution are bounded by zero and a constant $M$ :

$$
0<u, v \leq M
$$

3. Hölder continuity. Let us now turn to the question of Hölder continuity of weak solutions to (2.1) under assumptions (2.3)-(2.7). To establish Hölder continuity of weak solutions to problem $(2.1)-(2.12)$ it suffices to show that they belong to the classes $\mathfrak{B}_{2}(Q, M, \gamma, r, \delta, \kappa)$ (inequality (3.2)) and $\mathfrak{B}_{2}\left(Q \cup \partial Q^{\prime}, M, \gamma, r, \delta, \kappa\right)$ (inequality (3.3)) introduced in $[8, \S \S 7$ and 8 of Chapter II], with $M, \gamma, q, r, \delta, \kappa$ being fixed positive numbers and $\partial Q^{\prime}$ the part of the boundary $\partial Q$. Our main result in this section is the following 
THEOREM 3.1. Let $(u, v)$ be a solution to system (2.1) and let assumptions (2.2)-(2.9) be satisfied. Then

$$
(u, v) \in \mathfrak{B}_{2}(Q, M, C, r, \delta, \kappa) \quad \text { and } \quad(u, v) \in \mathfrak{B}_{2}\left(Q \cup \partial Q^{\prime}, M, C, r, \delta, \kappa\right) .
$$

For every $\varphi \in L^{1}(Q)$ and $0<h<T$ let us introduce the Steklov averages

$$
\varphi(x, t)_{h} \equiv \begin{cases}\frac{1}{h} \int_{t}^{t+h} \varphi(x, \tau) d \tau, & t \in(0, T-h], \\ 0, & t>T-h,\end{cases}
$$

for all $0<t<T$. Recall that for $\varphi \in L^{q}(\Omega \times(0, T))$ we have $\varphi_{h} \rightarrow \varphi$ in $L^{q}(\Omega \times(0, T-\varepsilon))$ as $h \rightarrow 0$ for every $\varepsilon \in(0, T)$; and for $\varphi \in C\left(0, T ; L^{q}(\Omega)\right)$, $\varphi_{h}(t) \rightarrow \varphi(t)$ in $L^{q}(\Omega)$ as $h \rightarrow 0$ for every $t \in(0, T-\varepsilon)$ and all $\varepsilon \in(0, T)$.

In order to prove interior regularity of solutions we need the following set of notations.

Let $K_{\varrho}$ be the $n$-dimensional open ball centered at $x_{0}$ of radius $\varrho$ contained in $\Omega$ :

$$
K_{\varrho} \equiv\left\{x \in \mathbb{R}^{n}|| x-x_{0} \mid<\varrho\right\} .
$$

Let $Q(\tau, \varrho)$ be a cylinder contained in $Q$ of height $\tau$ built up upon $K_{\varrho}$ :

$$
Q(\tau, \varrho) \equiv K_{\varrho} \times\left\{t_{0}, t_{0}+\tau\right\}=\left\{\left|x-x_{0}\right|<\varrho, t_{0}<t<t_{0}+\tau\right\} .
$$

Write

$$
(H-k)_{+}(x, t)=\max \{(H(x, t)-k), 0\} .
$$

Introduce a set of positive numbers $k$ subject to the condition

$$
\underset{Q(\varrho, \tau)}{\operatorname{ess} \sup }\left|(H(x, t)-k)_{+}\right| \leq \delta .
$$

Set

$$
A_{k, \varrho}(t) \equiv\left\{x \in K_{\varrho} \mid(H(x, t)-k)_{+}>0\right\},
$$

were $\varrho$ and $\tau$ are some positive numbers so small that $Q(\tau, \varrho) \subset Q$.

Let $\zeta(x, t)$ be a continuous, piecewise smooth cut-off function ranging from 0 to 1 and equal to zero on the lateral boundary of the cylinder $Q(\tau, \varrho)$. Set

$$
q=\frac{2(2+n)}{n} .
$$

In order to prove regularity up to the boundary we need some additional notations. $K_{\varrho}$ and $Q(\varrho, \tau)$ are no more fully contained in the domains $\Omega$ and $Q$.

We impose an additional condition upon the set of levels $k$ :

$$
k \geq \max _{Q(\varrho, \tau) \cap \partial Q^{\prime}} H(x, t) .
$$

Let

$$
B_{k, \varrho}(t) \equiv\left\{x \in K_{\varrho} \cap \Omega \mid(H(x, t)-k)_{+}>0\right\} .
$$


Proof of Theorem 3.1. Multiply the first equation of (2.1) by $H_{u}$ and add the second one multiplied by $H_{v}$ (the $H$ stands for $H_{1}$ or $H_{2}$ ). Choose $\left(H_{h}-k\right)_{+} \zeta^{2}(x, t)$ as a testing function. After integrating in $t$ from $t_{0}$ to $t$, $0 \leq t_{0} \leq t \leq T$, and in $x$ over the domain $\Omega$ and letting $h \rightarrow 0$, this results in

$$
\begin{aligned}
\frac{1}{2} \int_{\Omega}(H- & k)_{+}^{2} \zeta^{2}(x, t)+\iint_{\Omega \times\left(t_{0}, t\right)}\left\{\left\langlea_{1} \nabla u+b_{1} \nabla v, H_{u u}(H-k)_{+} \nabla u\right.\right. \\
& \left.+H_{u v}(H-k)_{+} \nabla v+H_{u}^{2} \nabla u+H_{u} H_{v} \nabla v\right\rangle \\
& +\left\langle a_{2} \nabla u+b_{2} \nabla v, H_{u u}(H-k)_{+} \nabla u\right. \\
& \left.\left.+H_{u v}(H-k)_{+} \nabla v+H_{u}^{2} \nabla u+H_{u} H_{v} \nabla v\right\rangle\right\} \chi_{A_{k, \varrho}} \zeta^{2} \\
& +2 \iint_{\Omega \times\left(t_{0}, t\right)}\left\langle\left(a_{1} H_{u}+a_{2} H_{v}\right) \nabla u+\left(b_{1} H_{u}+b_{2} H_{v}\right) \nabla v, \nabla \zeta\right\rangle \zeta(H-k)_{+} \\
\leq & \iint_{\Omega \times\left(t_{0}, t\right)} \frac{f_{1} H_{u}+f_{2} H_{v}}{\sqrt{1+|u|+|v|}}(H-k)_{+} \zeta^{2} \\
& +\frac{1}{2} \int_{\Omega}(H-k)_{+}^{2} \zeta^{2}\left(x, t_{0}\right)+\iint_{\Omega \times\left(t_{0}, t\right)}(H-k)_{+}^{2} \zeta \zeta_{t} .
\end{aligned}
$$

We have

$$
\begin{aligned}
&\left\langle a_{1} \nabla u+\right.\left.b_{1} \nabla v, H_{u u}(H-k)_{+} \nabla u+H_{u v}(H-k)_{+} \nabla v+H_{u}^{2} \nabla u+H_{u} H_{v} \nabla v\right\rangle \\
&+\left\langle a_{2} \nabla u+b_{2} \nabla v, H_{u u}(H-k)_{+} \nabla u+H_{u v}(H-k)_{+} \nabla v+H_{u}^{2} \nabla u+H_{u} H_{v} \nabla v\right\rangle \\
&=\left\{\left[a_{1} H_{u}^{2}+a_{2} H_{u} H_{v}\right]|\nabla u|^{2}+\left[\left(a_{1}+b_{2}\right) H_{u} H_{v}+b_{1} H_{u}^{2}+a_{2} H_{v}^{2}\right](\nabla u \nabla v)\right. \\
&+ \\
&\left.+\left[b_{1} H_{u} H_{v}+b_{2} H_{v}^{2}\right]|\nabla v|^{2}\right\}+\left\{\left[a_{1} H_{u u}+a_{2} H_{u v}\right]|\nabla u|^{2}\right. \\
&+\left[\left(a_{1}+b_{2}\right) H_{u v}+b_{1} H_{u u}+a_{2} H_{v v}\right](\nabla u \nabla v) \\
&\left.+\left[b_{1} H_{u v}+b_{2} H_{v v}\right]|\nabla v|^{2}\right\}(H-k)_{+} .
\end{aligned}
$$

According to hypotheses (2.6) the first curly brackets give

$$
\begin{aligned}
\{\ldots\} & =\Lambda(x, u, v) H_{u}^{2}|\nabla u|^{2}+\Lambda(x, u, v) H_{u} H_{v}(\nabla u \nabla v)+\Lambda(x, u, v) H_{v}^{2}|\nabla v|^{2} \\
& =\Lambda(x, u, v)|\nabla H(u, v)|^{2} .
\end{aligned}
$$

In virtue of hypothesis (2.7) for the second curly brackets we have

$$
\{\ldots\}(H-k)_{+} \geq 0 .
$$

We also have

$$
a_{1} H_{u}+a_{2} H_{v}=\Lambda H_{u}, \quad b_{1} H_{u}+b_{2} H_{v}=\Lambda H_{v} .
$$

Hence, making use of hypotheses (2.4), (2.5), Young's inequality, Hölder's 
inequality, and assumptions (2.2), we get

$$
\begin{gathered}
\int_{K_{\varrho}}(H-k)_{+}^{2} \zeta^{2}\left(x, t_{0}+\tau\right)+\left(\Lambda_{1} / 2\right) \iint_{Q(\tau, \varrho)}\left|\nabla(H-k)_{+}\right|^{2} \zeta^{2} \\
\leq \int_{K_{\varrho}}(H-k)_{+}^{2} \zeta^{2}\left(x, t_{0}\right)+C\left\{\iint_{Q(\tau, \varrho)}\left(|\nabla \zeta|^{2}+\zeta\left|\zeta_{t}\right|\right)(H-k)_{+}^{2}\right. \\
\left.+\left[\int_{t_{0}}^{t_{0}+\tau}\left|A_{k, \varrho}(t)\right|\right]^{2(1+\kappa) / q}\right\} .
\end{gathered}
$$

The case of $(-H-k)_{+}$is self-evident. According to [8] this proves that $H \in \mathfrak{B}_{2}(Q, M, C, q, \delta, \kappa)$. Considering the sets $K_{\varrho} \cap \Omega$ and $Q(\tau, \varrho) \cap Q$ we similarly get

$$
\begin{gathered}
\int_{K_{\varrho} \cap \Omega}(H-k)_{+}^{2} \zeta^{2}\left(x, t_{0}+\tau\right)+\left(\Lambda_{1} / 2\right) \quad \iint_{Q(\tau, \varrho) \cap Q}\left|\nabla(H-k)_{+}\right|^{2} \zeta^{2} \\
\leq \int_{K_{\varrho}}(H-k)_{+}^{2} \zeta^{2}\left(x, \max \left[0, t_{0}\right]\right)+C\left\{\iint_{Q(\tau, \varrho) \cap Q}\left(|\nabla \zeta|^{2}+\zeta\left|\zeta_{t}\right|\right)(H-k)_{+}^{2}\right. \\
\left.+\left[\int_{\max \left[0, t_{0}\right]}^{t_{0}+\tau}\left|B_{k, \varrho}(t)\right|\right]^{2(1+\kappa) / q}\right\} .
\end{gathered}
$$

According to [8] this proves that $H \in \mathfrak{B}_{2}\left(Q \cup \partial Q^{\prime}, M, C, q, \delta, \kappa\right)$. Hence $H_{1,2} \in H^{\alpha, \alpha / 2}(Q \cup \partial Q)$ with $\alpha$ depending only on the data. From the assumption (2.10) it follows that $(u, v) \in H^{\alpha, \alpha / 2}(Q \cup \partial Q)$.

Now let us show how to obtain Hölder continuity of $u$ and $v$ for the case described in the Example. Subtracting $H_{2}$ from $H_{1}$ we get $\left(k_{1}-k_{2}\right) v=$ $H_{1}-H_{2}$ and hence $v$ is Hölder continuous. From the expression for $H_{1}$ we get

$$
\begin{aligned}
\mid u\left(x^{\prime}, t^{\prime}\right)- & u(x, t)|-| k_{1}|| v\left(x^{\prime}, t^{\prime}\right)-v(x, t) \mid \\
& -|\delta|\left(\left|u\left(x^{\prime}, t^{\prime}\right)+u(x, t)\right|\left|u\left(x^{\prime}, t^{\prime}\right)-u(x, t)\right|\right. \\
& +\left|v\left(x^{\prime}, t^{\prime}\right)+v(x, t)\right|\left|v\left(x^{\prime}, t^{\prime}\right)-v(x, t)\right| \\
& \left.+|\varepsilon|\left|u\left(x^{\prime}, t^{\prime}\right)\right|\left|v\left(x^{\prime}, t^{\prime}\right)-v(x, t)\right|+|\varepsilon||v(x, t)|\left|u\left(x^{\prime}, t^{\prime}\right)-u(x, t)\right|\right) \\
\leq & \left|H_{1}\left(x^{\prime}, t^{\prime}\right)-H_{1}(x, t)\right| .
\end{aligned}
$$

Substituting here $\left|v\left(x^{\prime}, t^{\prime}\right)-v(x, t)\right| \leq C\left(\left|x^{\prime}-x\right|+\left|t^{\prime}-t\right|^{1 / 2}\right)^{\alpha}$ for $\left(x^{\prime}, t^{\prime}\right),(x, t)$ $\in Q$, and making use of the assumptions upon $\delta$ and $\varepsilon$, we get

$$
\left|u\left(x^{\prime}, t^{\prime}\right)-u(x, t)\right| \leq C\left(\left|x^{\prime}-x\right|+\left|t^{\prime}-t\right|^{1 / 2}\right)^{\alpha} \quad \text { for }\left(x^{\prime}, t^{\prime}\right),(x, t) \in Q .
$$

4. Full regularity. In this section we additionally assume that $\partial \Omega$ belongs to $C^{2, \alpha}$ and that 


$$
\begin{aligned}
g^{\mu} & \in B C^{2+\alpha_{g}, 1+\alpha_{g} / 2}(S), \\
u_{0}, v_{0} & \in B C^{2+\alpha_{0}}(\bar{\Omega} \times\{0\}), \\
f_{1}, f_{2} & \in B C^{\alpha_{f}, \alpha_{f} / 2}(Q),
\end{aligned}
$$

and there exists $\Lambda_{1}>0$ such that

$$
\begin{aligned}
&\left|a_{1}-\Lambda_{1}\right|,\left|b_{2}-\Lambda_{1}\right|,\left|b_{1}\right|,\left|a_{2}\right|,\left|a, b_{j u}\right|,\left|a, b_{j v}\right|,\left|a, b_{j u u}\right|,\left|a, b_{j u v}\right|,\left|a, b_{j v v}\right| \\
& \leq \beta= 2^{-7}(1+C)^{-1}\left(1+[u]_{\alpha ; Q}+[v]_{\alpha ; Q}\right)^{-1} \\
& \times\left(1+M^{2}+\left|\widetilde{u}_{0}\right|_{2+\alpha ; Q}^{2}+\left|\widetilde{v}_{0}\right|_{2+\alpha ; Q}^{2}+\left|f_{1}\right|_{\alpha ; Q}^{2}+\left|f_{2}\right|_{\alpha ; Q}^{2}\right)^{-1},
\end{aligned}
$$

where $C$ is the constant obtained from the constant in estimate (4.5) by equating in the latter the Hölder norms of the coefficients to zero.

Theorem 4.1 (Schauder estimates [9, Theorem 4.28]). Let u be a solution in $B C^{2+\alpha, 1+\alpha / 2}(Q)$ of a linear parabolic equation

$$
u_{t}+L(u)=f \quad \text { for }(x, t) \in Q
$$

satisfying $u=\widetilde{u}_{0}$ a.e. on $\partial Q$. Suppose that $\Omega$ is of class $C^{2, \alpha}, \alpha \in(0,1)$, the coefficients $a_{i j}, b_{i}, c$, and the right hand side $f$ belong to $B C^{\alpha, \alpha / 2}(Q)$, and the boundary values belong to $B C^{2+\alpha, 1+\alpha / 2}(Q)$. Then

$$
|u|_{2+\alpha ; Q} \leq C\left(\|u\|_{\infty}+|f|_{\alpha ; Q}+\left|\widetilde{u}_{0}\right|_{2+\alpha ; Q}\right),
$$

where the constant $C$ depends only on $n, \alpha$, the constants in the ellipticity condition, $\Omega$ and the norms of the coefficients of $L$ in $B C^{\alpha, \alpha / 2}(Q)$.

We make use of the following lemma.

Lemma 4.2. Let $\Omega$ be bounded domain, and $u \in C^{2, \alpha}(\bar{\Omega})(\alpha \in(0,1))$. Then for any $\varepsilon>0$,

$$
\begin{aligned}
& {[u]_{2} \leq \varepsilon[u]_{2+\alpha}+\widetilde{C}_{1}[u]_{0},} \\
& {[u]_{1} \leq \varepsilon[u]_{2+\alpha}+\widetilde{C}_{2}[u]_{0},}
\end{aligned}
$$

where $\widetilde{C}_{1}, \widetilde{C}_{2}$ depend on $n, \alpha, \Omega$, and $\varepsilon$.

For the proof see [3, Theorem 1.2, p. 18]. Also we use the following lemma (see [3, Lemma 1.1, p. 18]):

Lemma 4.3. Let $u, v \in C^{\alpha}(\bar{Q})$. Then

$$
[u v]_{\alpha} \leq[u]_{0}[v]_{\alpha}+[v]_{0}[u]_{\alpha} \leq|u|_{\alpha}|v|_{\alpha} .
$$

Theorem 4.4. Let $(u, v)$ be a solution to system (4.4) and let assumptions (4.1)-(4.4) be satisfied. Then

$$
\begin{aligned}
& |u|_{2+\alpha ; Q}+|v|_{2+\alpha ; Q} \leq C\left(\|u\|_{\infty}+\|v\|_{\infty}\right. \\
& \left.+\left|f_{1} \frac{1}{\sqrt{1+|u|+|v|}}\right|_{\alpha ; Q}+\left|f_{2} \frac{1}{\sqrt{1+|u|+|v|}}\right|_{\alpha ; Q}+\left|\widetilde{u}_{0}\right|_{2+\alpha ; Q}+\left|\widetilde{v}_{0}\right|_{2+\alpha ; Q}+1\right),
\end{aligned}
$$


where the constants $C$ and $\alpha$ depend only on the data. This implies the full regularity of the solution.

Proof. Let us rewrite system (2.1) in the form

$$
\left\{\begin{array}{l}
u_{t}+L_{1}(u, v)=f_{1} \frac{1}{\sqrt{1+|u|+|v|}}, \\
v_{t}+L_{2}(u, v)=f_{2} \frac{1}{\sqrt{1+|u|+|v|}}, \quad \text { a.e. }(x, t) \in Q .
\end{array}\right.
$$

Consider the approximation of the solution $(u, v)$ by smooth functions $\left(u^{k}, v^{k}\right) \in B C^{2+\alpha, 1+\alpha / 2}(Q)$ such that $\left(u^{k}, v^{k}\right) \rightarrow(u, v)$ as $k \rightarrow \infty$ a.e. in $Q$. Without loss of generality we may assume that $\left|u^{k}\right|_{2+\alpha ; Q} \geq\left|u^{k-1}\right|_{2+\alpha ; Q}$ and $\left|v^{k}\right|_{2+\alpha ; Q} \geq\left|v^{k-1}\right|_{2+\alpha ; Q}$. Adding and subtracting $u_{t}^{k}+L_{1}\left(u^{k}, v^{k}, u^{k-1}, v^{k-1}\right)$ and $u_{t}^{k}+L_{2}\left(u^{k}, v^{k}, u^{k-1}, v^{k-1}\right)$ respectively, to each equation of system $(2.1)$, we can see that every such approximation satisfies the system

$$
\left\{\begin{array}{c}
u_{t}^{k}+L_{1}\left(u^{k}, v^{k}, u^{k-1}, v^{k-1}\right) \\
=\left(u_{t}^{k}+L_{1}\left(u^{k}, v^{k}, u^{k-1}, v^{k-1}\right)-u_{t}-L_{1}(u, v)\right) \\
\quad+f_{1} \frac{1}{\sqrt{1+|u|+|v|}}, \\
\quad v_{t}^{k}+L_{2}\left(u^{k}, v^{k}, u^{k-1}, v^{k-1}\right) \\
=\left(v_{t}^{k}+L_{2}\left(u^{k}, v^{k}, u^{k-1}, v^{k-1}\right)-v_{t}-L_{2}(u, v)\right) \\
+f_{2} \frac{1}{\sqrt{1+|u|+|v|}}, \quad \text { a.e. }(x, t) \in Q,
\end{array}\right.
$$

where

$$
\begin{aligned}
& L_{1}\left(u^{k}, v^{k}, u^{k-1}, v^{k-1}\right)=a_{1}\left(u^{k-1}, v^{k-1}\right) \Delta u^{k}+b_{1}\left(u^{k-1}, v^{k-1}\right) \Delta v^{k} \\
&+ a_{1}\left(u^{k-1}, v^{k-1}\right)_{u} \nabla u^{k-1} \nabla u^{k-1}+a_{1}\left(u^{k-1}, v^{k-1}\right)_{v} \nabla v^{k-1} \nabla u^{k-1} \\
&+b_{1}\left(u^{k-1}, v^{k-1}\right)_{u} \nabla u^{k-1} \nabla v^{k-1}+b_{1}\left(u^{k-1}, v^{k-1}\right)_{v} \nabla v^{k-1} \nabla v^{k-1},
\end{aligned}
$$

and analogously for $L_{2}$.

From each equation of system (4.8) we extract the principle diagonal part $u_{t}^{k}-\Lambda_{1} \Delta u^{k}$ or $v_{t}^{k}-\Lambda_{1} \Delta v^{k}$ (by adding and subtracting $\Lambda_{1} \Delta u^{k}$ or $\Lambda_{1} \Delta v^{k}$ in each of the equations) and apply to it Theorem 4.1 treating the rest of the terms as the right hand side. After making use of the Young inequality, Lemma 4.2 and Lemma 4.3, we get for these approximations an estimate analogous to the estimate from Theorem 4.1:

$$
\begin{aligned}
&\left|u^{k}\right|_{2+\alpha ; Q}+\left|v^{k}\right|_{2+\alpha ; Q} \leq C\left(\left\|u^{k-1}\right\|_{\infty}^{2}+\left\|v^{k-1}\right\|_{\infty}^{2}\right. \\
&+\left|f_{1} \frac{1}{\sqrt{1+|u|+|v|}}\right|_{\alpha ; Q}+\left|f_{2} \frac{1}{\sqrt{1+|u|+|v|}}\right|_{\alpha ; Q}
\end{aligned}
$$




$$
\begin{aligned}
& +\left|u_{t}^{k}+L_{1}\left(u^{k}, v^{k}, u^{k-1}, v^{k-1}\right)-u_{t}-L_{1}(u, v)\right|_{\alpha ; Q} \\
& +\left|v_{t}^{k}+L_{2}\left(u^{k}, v^{k}, u^{k-1}, v^{k-1}\right)-v_{t}-L_{2}(u, v)\right|_{\alpha ; Q} \\
& \left.+\left.\widetilde{u_{0}^{k-1}}\right|_{2+\alpha ; Q}+\left|\widetilde{v_{0}^{k-1}}\right|_{2+\alpha ; Q}+\beta\left|u^{k-1}\right|_{2+\alpha ; Q}^{2}+\beta\left|v^{k-1}\right|_{2+\alpha ; Q}^{2}\right) .
\end{aligned}
$$

After using the above estimate recurrently we get on the right the sum

$$
\begin{aligned}
\mid u_{t}^{k}+ & L_{1}\left(u^{k}, v^{k}, u^{k-1}, v^{k-1}\right)-u_{t}-\left.L_{1}(u, v)\right|_{\alpha ; Q} \\
+\beta 2 \mid u_{t}^{k-1}+ & L_{1}\left(u^{k-1}, v^{k-1}, u^{k-2}, v^{k-2}\right)-u_{t}-\left.L_{1}(u, v)\right|_{\alpha ; Q} ^{2}+\cdots \\
& +\beta^{2^{k}-1} 2^{2^{k}-1}\left|u_{t}^{2}+L_{1}\left(u^{2}, v^{2}, u^{1}, v^{1}\right)-u_{t}-L_{1}(u, v)\right|_{\alpha ; Q}^{2^{k}}
\end{aligned}
$$

and analogously for $L_{2}$. Choose approximations $\left(u^{1}, v^{1}\right)$ and $\left(u^{2}, v^{2}\right)$ such that the term $\left|u_{t}^{2}+L_{1}\left(u^{2}, v^{2}, u^{1}, v^{1}\right)-u_{t}-L_{1}(u, v)\right|_{\alpha ; Q}$ in this sum is majorized by $1+\left|f_{1}\right|_{\alpha ; Q}$. The next approximations, $\left(u^{2}, v^{2}\right)$ and $\left(u^{3}, v^{3}\right)$, are still closer to $(u, v)$, so $\left|u_{t}^{3}+L_{1}\left(u^{3}, v^{3}, u^{2}, v^{2}\right)-u_{t}-L_{1}(u, v)\right|_{\alpha ; Q}$ is all the more less than $1+\left|f_{1}\right|_{\alpha ; Q}$. The next approximation is still closer to $(u, v)$, and so on. Thus $\left|u_{t}^{k}+L_{1}\left(u^{k}, v^{k}, u^{k-1}, v^{k-1}\right)-u_{t}-L_{1}(u, v)\right|_{\alpha ; Q}$ is less than $1+\left|f_{1}\right|_{\alpha ; Q}$, and we find that this sum forms a convergent series as $k \rightarrow \infty$. A similar result is valid for the sum with $L_{2}$ :

$$
\begin{aligned}
\mid u_{t}^{k}+ & L_{2}\left(u^{k}, v^{k}, u^{k-1}, v^{k-1}\right)-u_{t}-\left.L_{2}(u, v)\right|_{\alpha ; Q} \\
+\beta 2 \mid u_{t}^{k-1}+ & L_{2}\left(u^{k-1}, v^{k-1}, u^{k-2}, v^{k-2}\right)-u_{t}-\left.L_{2}(u, v)\right|_{\alpha ; Q} ^{2}+\cdots \\
& +\beta^{2^{k}-1} 2^{2^{k}-1}\left|u_{t}^{2}+L_{2}\left(u^{2}, v^{2}, u^{1}, v^{1}\right)-u_{t}-L_{2}(u, v)\right|_{\alpha ; Q}^{2^{k}} .
\end{aligned}
$$

According to (4.4) the iterated estimate yields a convergent series.

Choosing the first approximation such that $\left|u^{1}\right|_{2+\alpha ; Q}+\left|v^{1}\right|_{2+\alpha ; Q} \leq 1$ and passing to the limit in (4.9) gives the sought-for estimate.

5. Global existence. Given the estimates of $L^{\infty}$ norms of a solution $(u, v)$ we have the following consequence of $[1$, Proposition 4.3] and [2, Theorem 2].

COROLlary 5.1. Let $(u, v)$ be a classical solution of (2.1) with boundary conditions (2.12). If there is a bound for $(u, v)$ in $C^{\alpha, \alpha / 2}(\bar{Q})$ with $\alpha>n /(n+1)$, which may depend on $T$ and the data, then the unique solution exists globally in time.

From the results of Section 4 it is easy to check that the hypotheses of this theorem are satisfied and so the nonnegative weak solution of $(2.1)-(2.12)$ with conditions (2.2)-(2.10), (4.4) is in fact classical and global in time.

\section{References}

[1] H. Amann, Dynamic theory of quasilinear parabolic systems, III. Global existence, Math. Z. 202 (1989), 219-250. 
[2] H. Amann, Dynamic theory of quasilinear parabolic equations, II. Reaction-diffusion systems, Differential Integral Equations 3 (1990), 13-75.

[3] Y. Z. Chen and L. C. Wu, Second Order Elliptic Equations and Elliptic Systems, Amer. Math. Soc., Providence, RI, 1998.

[4] E. De Giorgi, Un esempio di estremali discontinue per un problema variazionale di tipo ellittico, Boll. Un. Mat. Ital. 1 (1968), 135-137.

[5] E. DiBenedetto, Degenerate Parabolic Equations, Springer, New York, 1993.

[6] L. Dung, Hölder regularity for certain strongly coupled parabolic systems, J. Differential Equations 151 (1999), 313-344.

[7] K. H. W. Küfner, Global existence for a certain strongly coupled quasilinear parabolic systems in population dynamics, Analysis 15 (1995), 343-357.

[8] O. A. Ladyzhenskaya, N. A. Solonnikov and N. N. Ural'tseva, Linear and Quasilinear Equations of Parabolic Type, Amer. Math. Soc., Providence, RI, 1968.

[9] G. M. Lieberman, Second Order Parabolic Differential Equations, World Sci., Singapore 1996 .

[10] M. A. Pozio and A. Tesei, Global existence of solutions for a strongly coupled quasilinear parabolic system, Nonlinear Anal. 12 (1990), 657-689.

[11] M. Wiegner, Global solutions to a class of strongly coupled parabolic systems, Math. Ann. 292 (1992), 711-727.

Institute for Condensed Matter Physics

National Academy of Sciences of Ukraine

1 Svientsitskii Street, 79011 Lviv, Ukraine

E-mail: port@icmp.lviv.ua

Received on 10.3.2008;

revised version on 21.4 .2008 
